\title{
Fabricating Genetically Engineered High-Power Lithium Ion Batteries Using Multiple Virus Genes
}

Yun Jung Lee, ${ }^{1 *}$ Hyunjung Yi, ${ }^{1 *}$ Woo-Jae Kim, ${ }^{2}$ Kisuk Kang, ${ }^{3,4}$ Dong Soo Yun, ${ }^{1}$ Michael S. Strano, ${ }^{2}$ Gerbrand Ceder, ${ }^{1}$ Angela M. Belcher ${ }^{1,5 \dagger}$

${ }^{1}$ Department of Materials Science and Engineering, Massachusetts Institute of Technology, Cambridge, MA 02139, USA. ${ }^{2}$ Department of Chemical Engineering, Massachusetts Institute of Technology, Cambridge, MA 02139, USA. ${ }^{3}$ Department of Materials Science and Engineering, Korea Advanced Institute of Science and Technology, 335, Gwahangno, Yuseong-gu, Daejeon, Korea, 305-701. ${ }^{4}$ KAIST Institute for Eco-Energy, 335, Gwahangno, Yuseong-gu, Daejeon, Korea, 305-701. ${ }^{5}$ Department of Biological Engineering, Massachusetts Institute of Technology, Cambridge, MA 02139, USA.

*These authors contributed equally to this work.

${ }^{\dagger}$ To whom correspondence should be addressed. E-mail: belcher@mit.edu

Development of materials that deliver more energy at high charge/discharge rates is important for high power applications including portable electronic devices and hybrid electric vehicles. Reducing materials dimensions for lithium ion batteries can boost $\mathrm{Li}^{+}$ion and electron transfer in nanostructured electrodes. We developed a strategy for attaching electrochemically active materials to conducting carbon nanotubes networks through biological molecular recognition. By manipulating twogenes of the M13 virus, viruses were equipped with peptide groups with affinity for single-walled carbon nanotubes (SWNTs) on one end and peptides capable of nucleating amorphous iron phosphate $\left(\mathrm{a}-\mathrm{FePO}_{4}\right)$ fused to the viral major coat protein. For the virus clone that demonstrated 10 times greater affinity towards SWNTs, power performance of a-FePO ${ }_{4}$ was comparable to that of crystalline lithium iron phosphate $\left(\mathrm{c}-\mathrm{LiFePO}_{4}\right)$. The electrodes showed excellent capacity retention upon cycling at $1 \mathrm{C}$ for at least 50 cycles. This environmentally benign low temperature biological scaffold could facilitate fabrication of electrodes from materials that have been excluded because of their extremely low electronic conductivity.

Lithium ion battery electrodes store and release electrical energy by insertion and extraction of $\mathrm{Li}^{+}$ions and electrons through the electrode materials. Therefore, increasing transport of $\mathrm{Li}^{+}$ions and electrons in electrodes can enhance energy storage at high charge and discharge rates. Controlling nanostructure has become a critical process in developing electrode materials to boost transport in composite electrodes $(1,2)$ especially for the electrically insulating transition metal phosphate cathode materials. Among them, iron phosphate based materials have elicited attention as promising $\mathrm{Li}$ ion battery positive electrode materials due to their lower toxicity, lower cost, and improved safety through improved chemical, thermal, and structural stability for high power applications (3). However, their practical use has been constrained due to kinetic limitations, which result in poor charge and discharge rate capability and fading of capacity upon prolonged cycling. To address the rate limitation of these materials, most researchers have focused on tailoring particle size $(4,5)$ to reduce both the ionic and electronic path within the particles and enhancing electronic conductivity with surface carbon coating layers (6) or conducting nanoparticles additives $(7,8)$. However, the fabrication of nanosized particles is still challenging since the materials require at least $350^{\circ} \mathrm{C}$ for crystallization and carbon coating. Despite the recent advances in novel synthesis, the smallest particle size remains 20-40 $\mathrm{nm}$ (5).

Biological systems offer capabilities for environmentally benign materials synthesis. An M13 virus-based biological toolkit has been developed for the design of nanoarchitectured structures and materials (9-12). Our group has shown that M13 bacteriophage (phage or virus) can be used for battery device fabrication with improved performance by synthesizing electrochemically active anode nanowires and organizing the virus on a polymer surface (11, 13). However, in designing nanostructured electrodes with better electrical wiring for high power batteries, multifunctionality of the virus is required. Multifunctional viruses have been engineered with desired modifications on different positions of the protein coat $(10,12,14)$. Here we demonstrate a genetically programmed multifunctional virus as a versatile scaffold for the synthesis and assembly of materials for high power batteries.

Virus-enabled nanostructured cathode materials were first demonstrated by templating amorphous anhydrous iron 
phosphate (a-FePO 4$)$ on the E4 virus. E4 is a modified M13 virus that has tetraglutamate (EEEE-) fused to the amino terminus of each copy of pVIII major coat protein. Due to the presence of extra carboxylic acid groups compared with wildtype M13 virus (M13KE), the E4 virus exhibits increased ionic interactions with cations, and can serve as a template for materials growth $(11,13,15)$. Since only one gene (gVIII in Fig. 2A) has been modified for the desired peptide motif on pVIII, we call this E4 clone a one-gene system. Amorphous iron phosphate nanowires were produced on silver nanoparticles (Ag NPs) loaded E4 virus. Details of the synthesis procedure are given in the Supporting Online Material (16). Uniformly distributed Ag NPs loading along the coat protein of E4 virus (fig. S1) was initially intended to increase electronic conductivity $(7,8,11)$. The chemical analysis by direct current plasma atomic emission spectroscopy (DCP-AES) confirmed the atomic ratio of Fe to $\mathrm{P}$ as 1: 1 . Although viruses themselves have phosphate groups in their DNA (7270 phosphate group per one virus particle), the fraction of phosphate groups from DNA is below $1 \%$. Figure 1A shows transmission electron microscope (TEM) images of a-FePO ${ }_{4}$ nanowires with particle size of 10-20 nm in diameter templated on the virus. Generally, hydrated a$\mathrm{FePO}_{4}\left(\mathrm{a}-\mathrm{FePO}_{4} \bullet \mathrm{nH}_{2} \mathrm{O}, \mathrm{n}=2-4\right)$ is precipitated in aqueous solutions containing $\mathrm{Fe}^{3+}$ and $\mathrm{PO}_{4}{ }^{3-}$ ions around $\mathrm{pH}=7-8$ and anhydrous structures can be obtained through the dehydration of a-FePO ${ }_{4} \cdot \mathrm{nH}_{2} \mathrm{O}$ by thermal annealing at $400{ }^{\circ} \mathrm{C}$. Most structural water in a-FePO${ }_{4} \bullet \mathrm{nH}_{2} \mathrm{O}$ is removed from the structure around $200{ }^{\circ} \mathrm{C}$ (17). Surprisingly the viral nanowires produced on Ag NPs-loaded E4 was anhydrous as synthesized as shown by thermo gravimetric analysis (TGA) (Fig. 1B, fig. S2). Without Ag NPs, nanowires have about 10 $\mathrm{w} \%$ structural water, which corresponds to $\mathrm{n}=1$ in a$\mathrm{FePO}_{4} \bullet \mathrm{nH}_{2} \mathrm{O}$. X-ray powder diffraction of a-FePO $\mathrm{P}_{4}$ nanowires on Ag NPs-loaded E4 (fig. S3A) showed only peaks indexed as silver chloride (AgCl). We speculate that the dehydration of $\mathrm{FePO}_{4} \bullet \mathrm{nH}_{2} \mathrm{O}$ is related to the chlorination of Ag NPs, which could occur during the incubation with the iron chloride precursor. Part of the chlorinated AgCl was reduced to metallic Ag after electrochemical test. (fig. S3B). The reduced metallic Ag could enhance local electronic conductivity as Au nanoparticles could in $\mathrm{Co}_{3} \mathrm{O}_{4} / \mathrm{Au}$ heterostructured nanowires (11). Although the exact mechanism of dehydration is under investigation, dehydration of structural water without thermal treatment was accomplished by low temperature and environmentally benign chemistry. The dehydrated structure increases the theoretical capacity to $178 \mathrm{mAh} / \mathrm{g}$ making it a good cathode material.

The electrochemical performance of viral a-FePO 4 nanowires as a lithium ion battery cathode was evaluated.

(Fig. 1, C and D) Positive electrodes were prepared by mixing viral a-FePO 4 with Super P (TIMCAL, SUPER P® Li) carbon black and polytetrafluoroethylene (PTFE) binder in a mass ratio of 70: 25: 5. Details of the weight ratio of components are given in the Supporting Online Material (16). The first discharge capacity at a low discharge rate of C/10 (18) was $165 \mathrm{mAh} / \mathrm{g}$ (93\% of the theoretical value) and that of $1 \mathrm{C}$ discharge rate was $110 \mathrm{mAh} / \mathrm{g}$ (Fig. 1C) (19). The rate performance is also presented as a Ragone plot (Fig. 1D). In most electrode materials, specific energy decreases substantially as one applies more power (high rates) drawing more current from the electrodes (20). These rate performance values are similar to the best reported values for a-FePO ${ }_{4}$ synthesized at high temperature (21). Even with this one gene system, the nanostructuring of a-FePO 4 nanowires by the virus enabled an enhanced performance. However, high power performance and capacity retention upon cycling of both the biologically and traditionally synthesized electrodes are still inferior to commercially available c$\mathrm{LiFePO}_{4}$ cathodes.

Since our particles were already $10-20 \mathrm{~nm}$ in diameter, our strategy for improved performance was to improve the electronic conductivity in the cathode by achieving better electrical contact between the active materials. Although metallic Ag nanoparticles can locally enhance the electronic conductivity, more important for improved high power performance is a percolating network throughout the electrodes. It is known that incorporation of well-dispersed materials with high conductivity and high aspect ratio leads to efficient percolating networks $(22,23)$. Carbon nanotubes (CNTs) have been shown to meet these needs (23), thus welldispersed single wall CNTs (SWNTs) in water were used. However, conventional composite electrode fabrication processes inevitably suffer from aggregation of carbon particles thereby diminishing contact with the active materials (23). To achieve better electrical wiring to our biologically derived a-FePO${ }_{4}$, a specific affinity was engineered between the conducting material and active material.

Since the major coat protein of the E4 virus serves only as a template for a-FePO ${ }_{4}$ nanowires growth, additional genetic modification was required to engineer the $\mathrm{E} 4$ virus to have a binding affinity for SWNTs. In this context, the p3 protein (pIII), a minor coat protein located at one end of the virus (Fig. 2A), is an ideal tool since gene III can be independently controlled from gene VIII to insert foreign DNA encoding pIII-displayed peptides. Moreover, the peptide sequences identified through the phage-display with a pIII phage-display library can be directly inserted into the E4 virus without losing functionality (12). Therefore, phage-display experiments to search for peptide sequences with a strong binding affinity for SWNTs were done first, and then genetic engineering into the $\mathrm{E} 4$ virus to produce a multifunctional virus structure (see Methods for details of the procedure 
(16)). Several consensus sequences were obtained from separate phage-display screening experiments. Among them, sequences N'-HGHPYQHLLRVL-C', named as MC\#1, and N'-DMPRTTMSPPPR-C', as MC\#2, were selected for further experiments. The sequence MC\#1 started with histidine $(\mathrm{H})$, whose appearance in the first position was often observed in CNTs-binding sequences (24). Also it contained several aromatic residues ( $\mathrm{H}$ and $\mathrm{Y}$ ), which were expected to favorably bind to graphene surface via $\pi$-stacking interaction (25). The sequence MC\#2 is quite different from MC\#1 and the clone MC\#2 showed approximately four times higher binding affinity than the clone MC\#1 whose binding affinity is already two and a half times higher than wild-type M13KE in the binding affinity tests (26). (fig. S4, A and B) The strong binding of the sequence MC\#2 can be explained by the location of the hydrophobic segments of the sequence. The calculated hydrophobicity plot (fig. S5) shows a tri-block structure with hydrophilic regions on both ends and the hydrophobic region in the middle of the sequence. It has been demonstrated that a tri-block structure of hydrophilic/hydrophobic/hydrophilic polymers worked well to suspend SWNTs $(24,27)$.

To genetically engineer E4 virus as a multifunctional biological platform, the selected sequences, MC\#1 and MC\#2, were independently fused onto the amino-terminal portion of the pIII of E4 virus, producing clones EC\#1 and EC\#2 respectively (16). Since two genes (gIII and gVIII in Fig. 2A) have been engineered with desired modification on both pIII and pVIII proteins, we called it a two-gene system.

A schematic diagram for constructing the genetically engineered high power lithium ion battery using the multifunctional two-gene virus system is illustrated in Fig. 2B. All viruses were loaded with Ag NPs to synthesize anhydrous a-FePO . Formation of anhydrous a- $-\mathrm{FePO}_{4}$ on the pVIII preceded the interaction with SWNTs. The synthesis procedure of anhydrous a- $\mathrm{FePO}_{4}$ nanowires on the multifunctional viruses was the same for the growth on the one-gene system. Viral a- $\mathrm{FePO}_{4}$ solutions were then incubated with the SWNT suspensions to form a$\mathrm{FePO}_{4} / \mathrm{SWNTs}$ hybrid nanostructures (16). The photograph in Fig. 2B is the actual assembled lithium ion battery powering a light emitting diode (LED) using Li metal as a negative electrode. The virus-enabled high power battery could power a green LED with a small amount of active materials loading of $3.21 \mathrm{mg} / \mathrm{cm}^{2}$. While this cell was assembled with lithium foil as negative electrode, we have successfully made full virus-based $3 \mathrm{~V}$ batteries with various negative electrode materials (fig. S6).

The morphology of hybrid a-FePO $4 / \mathrm{SWNTs}$ nanowires on the EC\#2 virus is shown in Fig. 3 C-E. In the high-resolution TEM image (Fig. 3E), 6 8 SWNTs are bundled with diameters of $4-5 \mathrm{~nm}$. The TEM images show that a-FePO${ }_{4}$ nanowires templated on the multifunctional virus were tethered to SWNTs mainly through the pIII attachment, however, they made multiple contacts with neighboring SWNTs due to close positioning. To explore the effect of specificity, we also mixed the one-gene system (E4) viral nanowires solution with SWNTs. Most viral a-FePO nanowires on E4 did not make contact with SWNTs, and even if they did, the contact did not seem to be specific binding with SWNTs (fig. S7). Moreover, SWNTs aggregated by themselves when there was no specific binding affinity on pIII, suggesting that SWNTs specific viruses enhanced dispersion of SWNTs in solution. Similar observation has been reported showing that SWNTs specific peptides can disperse SWNTs while non-specific pepetides cannot (24).

The electrochemical properties of viral a-FePO ${ }_{4} /$ SWNTs hybrid materials with $5 \mathrm{wt} \%$ SWNTs were evaluated and compared. (Fig. 4) Positive electrodes were prepared by mixing viral a-FePO$/ 4$ SWNTs hybrid composites with Super $\mathrm{P}{ }^{\circledR}$ carbon black and PTFE binder in a mass ratio of 90: 5: 5 (16). The addition of $5 \mathrm{wt} \%$ extra carbon was used to increase the total volume making the powder easier to handle. Without extra carbon, the electrodes showed slightly higher polarization at high rates, but the difference was not substantial. As demonstrated in the first discharge profiles (Fig. 4A and fig. S8A for full discharge/charge curves) (19), electrochemical performances show remarkable improvement as the binding affinity to the SWNTs increases. Specific capacity at a low discharge rate of C/10 increased from 143 $\mathrm{mAh} / \mathrm{g}(\mathrm{E} 4)$ to $160 \mathrm{mAh} / \mathrm{g}$ with EC\#1 and to $170 \mathrm{mAh} / \mathrm{g}$ with EC\#2. The performance improvement is more pronounced at higher rates. Discharge profiles of the two-gene system show much lower polarization and maintain much higher capacity than those of the one-gene system at high rates. When compared with the best reported capacity for a-FePO 4 at a high rate of 3C ( $80 \mathrm{mAh} / \mathrm{g})$ (21), EC\#2 showed a capacity of $134 \mathrm{mAh} / \mathrm{g}$ confirming substantially improved high power performance. Moreover, when we cycled EC\#2 between 1.5 and $4.3 \mathrm{~V}$, the first discharge capacity at $10 \mathrm{C}$ reached 130 $\mathrm{mAh} / \mathrm{g}$. No published data for a-FePO${ }_{4}$ is available to compare with at a rate higher than $3 \mathrm{C}$, but this capacity value obtained for the two-gene system is comparable to the capacity from state-of-the-art c-LiFePO 4 . The power performance of the multifunctional virus based cathode was further compared using a Ragone plot. Figure 4B shows that two-gene system based materials delivered much higher energy than the one-gene system at high power. At a specific power of $4000 \mathrm{~W} / \mathrm{kg}$ (approximately corresponds to a rate of $10 \mathrm{C}$ ), the energy density of EC\#1 and EC\#2 was two times and three times higher respectively than that of E4. Again, the high power performance scales with binding affinity. In Fig. 4B inset, rate performance of E4 virus based cathodes with 
either Super P carbon or SWNTs were tested. It is well known that well-dispersed SWNTs by themselves make better electrical wiring to active materials due to better percolation networks than carbon black powders (23), confirming the importance of nanoscale electrical wiring. Figure 4C shows stable capacity retention of a$\mathrm{FePO}_{4} / \mathrm{SWNTs}$ hybrid electrodes upon cycling at $1 \mathrm{C}$. Up to 50 cycles, there was virtually no capacity fade observed. A slight capacity loss after the first cycle is a characteristic of a$\mathrm{FePO}_{4}$ materials $(17,21)$. When cycled at $\mathrm{C} / 10$ rate again after the sample was tested for several cycles at rates from $\mathrm{C} / 10$ to $10 \mathrm{C}$, the original capacity was recovered confirming structural stability (fig. S8B). Structural stability of viral a$\mathrm{FePO}_{4} / \mathrm{SWNTs}$ hybrid nanostructures was induced by materials specific binding and stiff/robust carbon nanotubes and brought about excellent retention at low SWNTs content of $5 \mathrm{wt} \%$. Since the density of SWNTs is $1.33 \mathrm{~g} / \mathrm{cm}^{3}$, it would decrease the volumetric energy density of the hybrid electrodes. However, although we adopted SWNTs to show that we can do nanoscale wiring by using genetic engineering, we expect that we could optimize the fraction of the conducting additives by using even better conducting nanowires with high aspect ratio and higher density.

There have been efforts to electrically address electrode materials with poor electronic conductivity through nanoscale wiring of active materials $(8,28,29)$. However, the wiring tools used so far were functionalized for a single component, either active materials $(8,29)$ or conducting materials $(28)$. The wiring did not completely exploit specificity but depended on random occurrence of contacts between either conducting networks or active materials. By developing this two-gene system with a universal handle to pick up electrically conducting carbon nanotubes, we facilitated a method to realize nanoscale electrical wiring for high power lithium ion batteries using basic biological principles. This biological scaffold could further extend possible sets of electrode materials by activating classes of materials that have been excluded because of their extremely low electronic conductivity. This versatile platform could also be conjugated with Li-containing positive electrodes, which is underway in our lab.

\section{References and Notes}

1. P. G. Bruce, B. Scrosati, J. M. Tarascon, Angewandte Chemie-International Edition 47, 2930 (2008).

2. M. Armand, J. M. Tarascon, Nature 451, 652 (2008).

3. S. Y. Chung, J. T. Bloking, Y. M. Chiang, Nature Materials 1, 123 (2002).

4. C. Delacourt, P. Poizot, S. Levasseur, C. Masquelier, Electrochemical and Solid State Letters 9, A352 (2006).

5. D. H. Kim, J. Kim, Electrochemical and Solid State Letters 9, A439 (2006).

6. J. M. Tarascon et al., Dalton Transactions, 2988 (2004).
7. F. Croce et al., Electrochemical and Solid State Letters 5, A47 (2002).

8. Y. S. Hu et al., Advanced Materials 19, 1963 (2007).

9. S. R. Whaley, D. S. English, E. L. Hu, P. F. Barbara, A. M. Belcher, Nature 405, 665 (2000).

10. K. T. Nam, B. R. Peelle, S. W. Lee, A. M. Belcher, Nano Letters 4, 23 (2004).

11. K. T. Nam et al., Science 312, 885 (2006).

12. Y. Huang et al., Nano Letters 5, 1429 (2005).

13. K. T. Nam et al., Proceedings of the National Academy of Sciences of the United States of America 105, 17227 (2008).

14. A. S. Khalil et al., Proceedings of the National Academy of Sciences of the United States of America 104, 4892 (2007).

15. K. T. Nam, Y. J. Lee, E. M. Krauland, S. T. Kottmann, A. M. Belcher, Acs Nano 2, 1480 (2008).

16. Materials and methods are available as supporting material on Science Online.

17. P. P. Prosini et al., Journal of the Electrochemical Society 149, A297 (2002).

18. Rates are reported in C-rate convention, where $C / n$ is the rate (current per gram) corresponding to complete charging or discharging to the theoretical capacity of the materials in $\mathrm{n}$ hours. Here, $1 \mathrm{C}$ corresponds to $178 \mathrm{~mA} / \mathrm{g}$.

19. In the rate test, the cell was charged at $\mathrm{C} / 10$ rate to $4.3 \mathrm{~V}$, and then held at $4.3 \mathrm{~V}$ until the current density was lower than $\mathrm{C} / 100$ and discharged at different rates.

20. K. S. Kang, Y. S. Meng, J. Breger, C. P. Grey, G. Ceder, Science 311, 977 (2006).

21. Z. C. Shi et al., Electrochimica Acta 53, 2665 (2008).

22. S. Ahn, Electrochemical and Solid State Letters 1, 111 (Sep, 1998).

23. J. S. Sakamoto, B. Dunn, Journal of the Electrochemical Society 149, A26 (2002).

24. S. Q. Wang et al., Nature Materials 2, 196 (2003).

25. M. Zheng et al., Science 302, 1545 (2003).

26. E. Ryan, US patent (2002).

27. V. C. Moore et al., Nano Letters 3, 1379 (2003).

28. L. Kavan, Chemistry of Materials 19, 4716 (2007).

29. Q. Wang, N. Evans, S. M. Zakeeruddin, I. Exnar, M. Gratzel, Journal of the American Chemical Society 129, 3163 (2007).

30. This work was supported by the Army Research Office Institute of the Institute of Collaborative Biotechnologies (ICB) and US National Science Foundation through the Materials Research Science and Engineering Centers programme. H. YI is grateful for Korean Government Overseas Scholarship. W-J.K. is grateful for support by the Korea Research Foundation Grant funded by the Korean Government (MOEHRD) (KRF-2005-214-D00260).K. K is grateful for the funding supported by Korea Science and 
Engineering Foundation of the Ministry of Education, Science and Technology (No. R01-2008-000-10913-0) and Energy Resource Technology Development program by the Ministry of Knowledge Economy (No. 2008-E-EL11P-08-3-010).M.S.S. is grateful for funding from the National Science Foundation, and the Office of Naval Research Young Investigator Grant

\section{Supporting Online Material}

www.sciencemag.org/cgi/content/full/1171541/DC1

Materials and Methods

Figs. S1 to S8

References

28 January 2009; accepted 25 March 2009

Published online 2 April 2009; 10.1126/science.1171541

Include this information when citing this paper.

Fig. 1. Characterization of a-FePO 4 nanowires cathodes in a one-gene viral system (E4). (A) TEM images of templated a$\mathrm{FePO}_{4}$ nanowires on E4 viruses. Inset: Magnified images of the same nanowires. (B) TGA curve of a-FePO 4 nanowires synthesized on Ag NPs loaded E4. For comparison, TGA curve of a-FePO${ }_{4} \cdot \mathrm{H}_{2} \mathrm{O}$ grown on E4 virus (without Ag NPs) is also presented. (C)-(D) Electrochemical performance of a$\mathrm{FePO}_{4}$ viral nanowires on E4 tested between 2.0 and $4.3 \mathrm{~V}$. Active materials loading was $2.63 \mathrm{mg} / \mathrm{cm}^{2}$. (C) First discharge curves at different rates. (D) The Ragone plot representing rate performance in terms of specific power vs specific energy (only active electrode mass included in the weight).

Fig. 2. Biological toolkits: genetic engineering and biomolecular recognition. (A) A schematic presentation of the multifunctional M13 virus is shown with its important proteins genetically engineered in this study. The gene VIII protein (pVIII), a major capsid protein of the virus, is modified to serve as a template for a-FePO $\mathrm{P}_{4}$ growth and the gene III protein (pIII) is further engineered to have a binding affinity for single-walled carbon nanotubes (SWNTs). (B) A schematic diagram for fabricating genetically engineered high power lithium ion battery cathodes using multifunctional viruses (two-gene system) and a photograph of actual battery used to power a green light-emitting diode (LED). The biomolecular recognition and attachment to conducting SWNT networks make efficient electrical nanoscale wiring to the active nanomaterials enabling high power performance. These hybrid materials were assembled as a positive electrode in a lithium ion battery using lithium metal foil as a negative electrode to power a green LED. Active cathode materials loading was $3.21 \mathrm{mg} / \mathrm{cm}^{2}$. The 2016 coin cell which is $2 \mathrm{~cm}$ in diameter and $1.6 \mathrm{~mm}$ in thickness was used. LED power dissipation was $105 \mathrm{~mW}$.
Fig. 3. Morphology of the a-FePO 4 grown on the multifunctional viruses/SWNTs hybrid nanostructures. TEM images of the following. (A) a-FePO 4 nanowires templated on EC\#2 viruses (before interacting with SWNTs). EC\#2 virus is a two-gene system virus with the strongest binding affinity to SWNTs. (B) SWNTs only (before interacting with viral a$\mathrm{FePO}_{4}$ ). (C)-(E), a-FePO 4 grown on EC\#2 attached to SWNTs. (C) Low magnification (10,000). (D) Higher magnification (30,000). (E) High resolution TEM (HRTEM) images $(800,000)$. For HRTEM imaging, surfactants were removed by washing with acetone. Material specific tethering of the viral a-FePO 4 to the SWNTs is visualized. Amorphous nature of $\mathrm{FePO}_{4}$ was also confirmed.

Fig. 4. Electrochemical properties of the a-FePO viral nanowires in two-gene systems tested between 2.0 and $4.3 \mathrm{~V}$. Rate capabilities and capacity retention upon cycling of the a$\mathrm{FePO}_{4}$ nanowires/SWNTs hybrid electrodes templated on different clones are presented. All a-FePO $/$ SWNTs hybrid materials had 5 w\% SWNTs. EC\#2 virus is a two-gene system virus with the strongest binding affinity to SWNTs, EC\#1 is a two-gene system virus with moderate binding affinity and E4 is one-gene system virus with no insert on pIII. (A) First discharge curves at different rates. Active materials loading were E4: $2.34 \mathrm{mg} / \mathrm{cm}^{2}, \mathrm{EC \# 1:} 2.31 \mathrm{mg} / \mathrm{cm}^{2}$, EC\#2: $2.62 \mathrm{mg} / \mathrm{cm}^{2}$. (B) Ragone plot showing improvement in high power performance with higher binding affinity towards SWNTs (only active electrode mass included in the weight). Inset: Comparison of rate capability of E4 virus based cathodes with either Super $\mathrm{P} \AA$ carbon or SWNTs. Electrode with well-dispersed SWNTs even with much smaller amount exhibited improved rate performance due to better percolation networks than carbon black powders. (C) Capacity retention for 50 cycles at $1 \mathrm{C}$ rate. There was no obvious fading for at least 50 cycles. Active materials loading were E4: $2.90 \mathrm{mg} / \mathrm{cm}^{2}$, EC\#1: $2.22 \mathrm{mg} / \mathrm{cm}^{2}$, EC\#2: 2.27 $\mathrm{mg} / \mathrm{cm}^{2}$. 


\section{$\mathbf{A}$}

Genes to be engineered

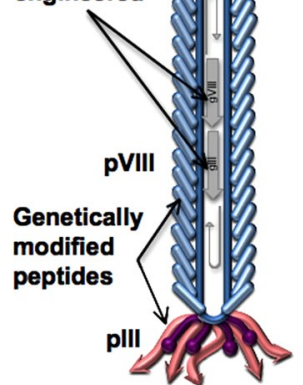

High Power Lithium Ion Battery Cathode

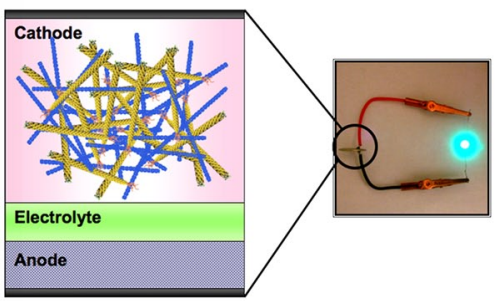




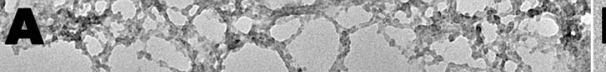

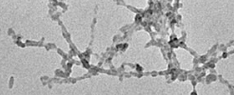

\section{3.

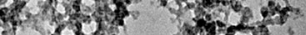

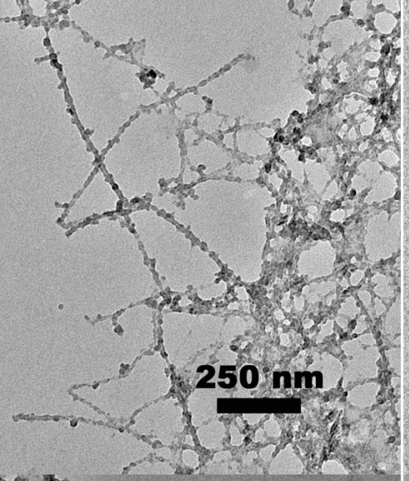

$24=5$ का

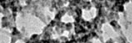

(2)

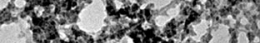

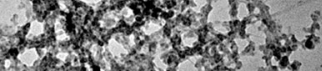

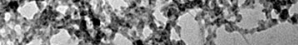

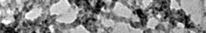

12 .

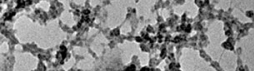

No the

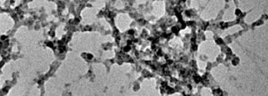

8

not of?

-

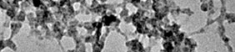
of 3 .

the or pro:
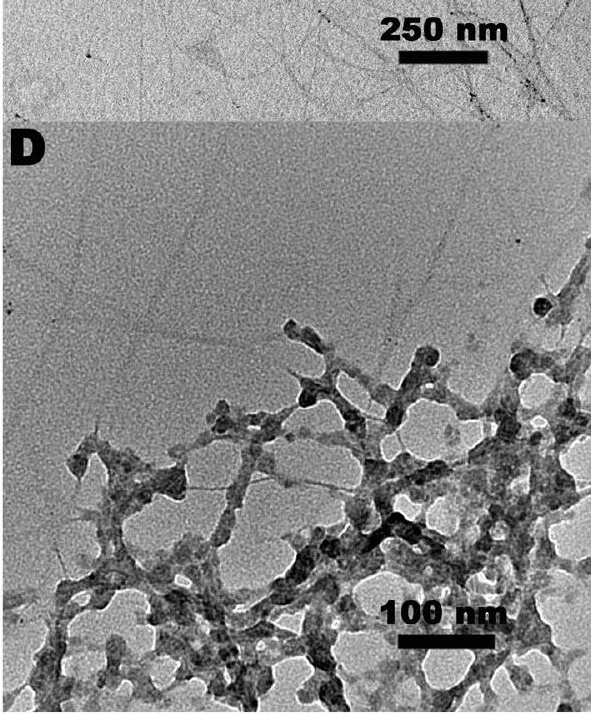


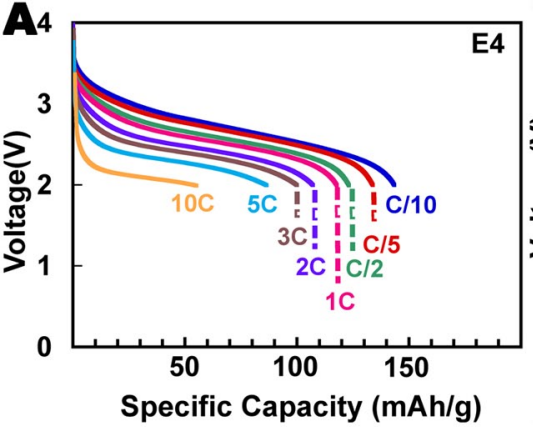

B

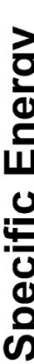

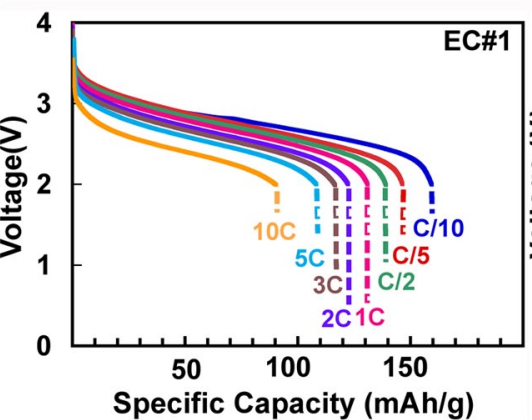

Specific Capacity (mAh/g)
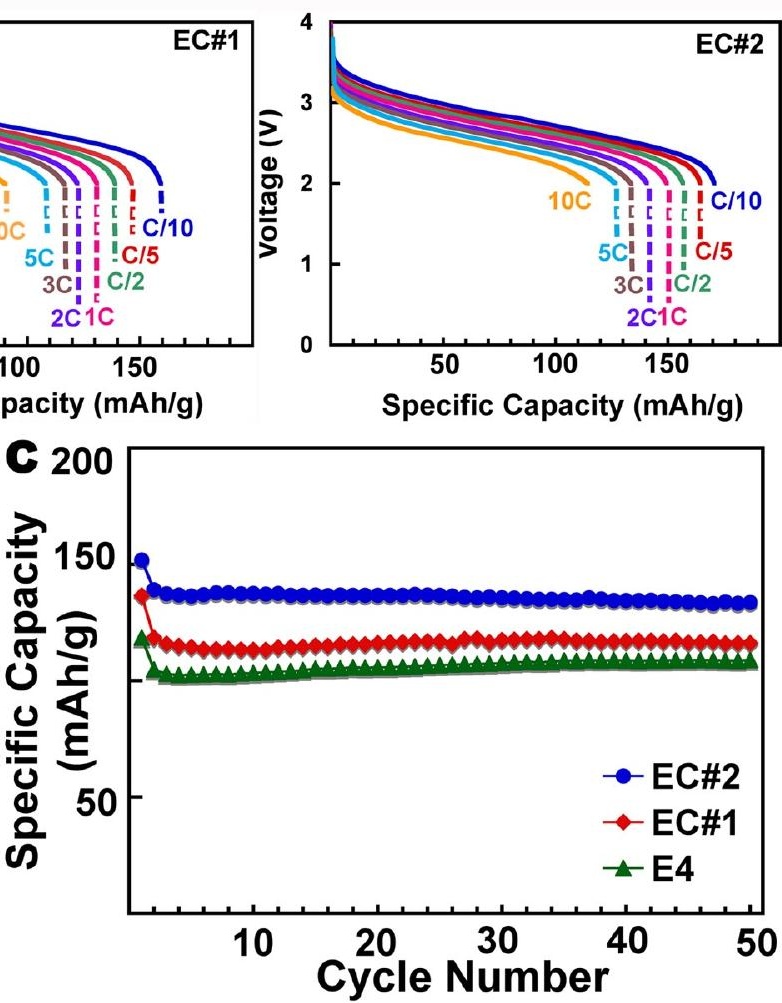